\title{
Cytomegalovirus Genotype and Virulence in Infants with Congenital Infection
}

\author{
Hong-bo $\mathrm{Hu}^{1}$ Jian-gang $\mathrm{Wu}^{2}$ Jian-jun Sun ${ }^{2}$ Qiao-ying Peng ${ }^{3}$ Xiao-peng Shang ${ }^{4}{ }^{\circ}$
}

\footnotetext{
1 Department of Laboratory, Maternal and Child Health Hospital of Hubei Province, People's Republic of China

2 Department of Laboratory, First People's Hospital of Guangshui, People's Republic of China

${ }^{3}$ Department of Neonatology, Maternal and Child Health Hospital of Hubei Province, People's Republic of China

${ }^{4}$ Department of Infectious Disease, First People's Hospital of

Guangshui, People's Republic of China
}

J Pediatr Infect Dis 2021;16:171-177.

\begin{abstract}
Address for correspondence Xiao-peng Shang, MD, Department of Infectious Disease, First People's Hospital of Guangshui, Guangshui, Hubei 432700, China (e-mail: xiaopengs0910@163.com).
\end{abstract}

\author{
Abstract \\ Keywords \\ - CMV genotypes \\ - symptomatic CMV \\ disease \\ - virulence \\ - congenital infection
}

Objective Cytomegalovirus (CMV) virulence may depend on genetic variability in several regions of the genome. This study aimed to assess specific CMV genotypes' association with the severity of symptomatic congenital CMV disease at birth.

Methods CMV glycoprotein B $(\mathrm{gB})$, glycoprotein $\mathrm{N}(\mathrm{gN})$, glycoprotein $\mathrm{H}(\mathrm{gH})$, and UL144 strains were identified by nested polymerase chain reaction, restriction fragment length polymorphism, and heteroduplex mobility assay single-stranded conformation polymorphism in 50 infants infected congenitally and 25 asymptomatic infants.

Results gN1 $(p=0.010)$ and UL144-B $(p=0.034)$ genotypes were associated, by logistic regression, with reduced risk of developing symptomatic congenital CMV infection. gN1 $(p=0.020)$ and gN3 $(p=0.022)$ genotypes were associated with reduced risk of severe symptomatic disease. Conversely, gB1 $(p=0.018)$ was the most virulent genotype and was associated with severe symptoms.

Conclusion An association among gB1, gN1, gN3, and UL144-B genotypes of CMV and severity of congenital CMV disease might exist. gB, gN, and UL144 genotypes could be important virological markers of infant infection.

\section{Introduction}

Some infants develop symptoms, sometimes severe, from congenital cytomegalovirus (CMV) infection. The host immune system affects the outcome of infection to some extent, but the CMV strain sequence polymorphisms may also shape outcomes and tissue tropism. ${ }^{1}$ Several envelope glycoproteins, encoded by UL55, UL73, and UL75, including glycoprotein $\mathrm{B}(\mathrm{gB})$, glycoprotein $\mathrm{N}(\mathrm{gN})$, and glycoprotein $\mathrm{H}$ $(\mathrm{gH})$, respectively, have been evaluated in clinical CMV iso- lates because of their influence on tissue tropism and virulence. ${ }^{2-4}$ The UL144 polymorphisms are concentrated in the $5^{\prime}$ end of the gene, especially in cysteine-rich domain 1 (CRD1), that may bind the B- and T-lymphocyte attenuator proteins. This domain's activity may vary among polymorphisms, leading to a range of clinical symptoms and prognoses. $^{5-7}$ Genetic polymorphisms of all these genes can be used to classify CMV strains and may affect the infectivity or pathogenicity of CMV. received

October 15, 2020 accepted after revision March 8, 2021

published online

June 15, 2021
DOI https://doi.org/ 10.1055/s-0041-1728743. ISSN 1305-7707.

\footnotetext{
(C) 2021. The Author(s).

This is an open access article published by Thieme under the terms of the Creative Commons Attribution-NonDerivative-NonCommercial-License, permitting copying and reproduction so long as the original work is given appropriate credit. Contents may not be used for commercial purposes, or adapted, remixed, transformed or built upon. (https://creativecommons.org/ licenses/by-nc-nd/4.0/) Georg Thieme Verlag KG, Rüdigerstraße 14, 70469 Stuttgart, Germany
} 
Few validated risk factors are available for outcome prediction or response to treatment, and little progress has been made in the treatment of infants with congenital disease. Additional research on congenital CMV infection should be focused on carefully designed randomized trials and multicenter prospective cohort studies. To this end, the $\mathrm{gB}, \mathrm{gN}, \mathrm{gH}$, and UL144 genotypes of CMV strains isolated from different clinical specimens of infants with symptomatic congenital CMV infection were studied, focusing on viral genes involved in virulence. The incidence of CMV genotypes was determined to serve as a baseline for direct comparison and support epidemiological research.

\section{Materials and Methods}

Infants at two hospitals with positive CMV infection and treated between January 2017 and December 2019 were included in the study. Demographical and clinical data were recorded, including gender, gestational age at birth, clinical signs, presence of abnormal imaging findings by ultrasound (US) or magnetic resonance (MR) compatible with CMV infection, neurologic abnormalities, failed hearing screen or proven sensorineural hearing loss (SNHL), chorioretinitis, and laboratory findings. Patients suffering from Epstein-Barr virus or other viral infections were excluded.

Symptomatic congenital CMV disease at birth is defined as the presence of at least one of the following conditions: (1) abnormal physical examination; (2) abnormal imaging findings by US or MR; (3) neurologic abnormalities; (4) abnormal visual examination; and (5) laboratory abnormalities., Asymptomatic congenital CMV infection is defined as no apparent abnormalities to suggest congenital CMV disease and normal hearing. Moderately to severely symptomatic CMV disease is defined as multiple manifestations attributable to CMV infection, including thrombocytopenia, petechiae, hepatomegaly, splenomegaly, intrauterine growth restriction, hepatitis (raised transaminases or bilirubin), or central nervous system involvement such as microcephaly, radiographic abnormalities consistent with CMV central nervous system disease, abnormal cerebrospinal fluid (CSF) indices for age, chorioretinitis, SNHL, or the detection of CMV DNA in CSF. Mildly symptomatic CMV disease is defined as an occurrence with one or two isolated manifestations of CMV infection that are mild and transient (e.g., mild hepatomegaly or a single measurement of low platelet count or raised levels of alanine aminotransferase). ${ }^{9}$

Patients were detected for CMV infection using serological tests (immunoglobulin M [IgM]), viral culture, and realtime polymerase chain reaction (PCR) for blood, urine, or CSF samples or a combination of these media. CMV IgM was assayed with an enzyme-linked immunosorbent assay kit following the manufacturer's instructions (DiaSorin S.p.A., Italy). Urine samples were collected and cultured using the shell vial culture method (Chemicon, Temecula, California, United States). A quantitative fluorescence CMV-DNA kit was used to assess CMV-DNA following the manufacturer's instructions (Daan Gene Company of Zhongshan University, China). DNA level $>10^{3}$ copies $/ \mathrm{mL}$ indicated the presence of viral genome and was considered positive. $\mathrm{CMV}$ gB, gN, gH, and UL144 genotypic analyses were used by nested PCR, restriction fragment length polymorphism, and heteroduplex mobility assay single-stranded conformation polymorphism, as previously reported ${ }^{10-13}$ (-Table $\mathbf{1}$ ).

Statistical analyses used SPSS version 21.0 software (SPSS, Inc., Chicago, Illinois, United States). Genotype distribution among congenitally infected patients, relationships among the $\mathrm{gB}, \mathrm{gN}, \mathrm{gH}$, and UL144 genotypes, and severity of CMV disease were analyzed using chi-square test for ratio comparisons. Logistic regression analysis was used to assess risk for symptomatic diseases or severity of symptomatic CMV diseases associated with particular genotypes. Multiple comparisons between the mild and moderate to severe

Table 1 Methodological approaches to discriminate gB, gN, gH, and UL144 genotypes

\begin{tabular}{|l|l|l|l|}
\hline Genotype & Method & Primers & Restriction endonucleases \\
\hline $\mathrm{gB}$ & PCR-RFLP & $\begin{array}{l}\text { External primers: 5'-GGC ATC AAG CAA AAA TCT-3' } \\
\text { Antisense: 5'-CAG TTG ACC GTA CTG CAC-3' } \\
\text { Internal primers: 5'-TGG AAC TGG AACGTT TGG C-3' } \\
\text { Antisense: 5'-GAA ACG CGC GGC AAT CGG-3' }\end{array}$ & $\begin{array}{l}\text { Hin f I } \\
\text { Rsa I }\end{array}$ \\
\hline $\mathrm{gN}$ & PCR-RFLP & $\begin{array}{l}\text { UL73-105725F: (5'-GGC GGT GGT GTG ATG GAG -3') } \\
\text { UL73-106122R: (5'-TTC TGG AAG CAG CAA TGT CG -3' }\end{array}$ & $\begin{array}{l}\text { Scal } \\
\text { Sall } \\
\text { Sacl }\end{array}$ \\
\hline $\mathrm{gH}$ & PCR-RFLP & $\begin{array}{l}\text { External primers: 5'-AGG TAT TGA CAG ATC AAT GG-3' } \\
\text { Antisense: 5'-CTC CTT CTC TCG GGT GTA AC-3' } \\
\text { Internal primers: 5'-TGG TGT TTT CAC GCA GGA A-3' } \\
\text { Antisense: 5'-CCA CCT GGA TCA CGC CGC TG-3' }\end{array}$ & Hhal \\
\hline UL144 & HMA-SSCP & $\begin{array}{l}\text { External primers: 5'-TCG TAT TAC AAA CCG CGG AGA GGA T-3' } \\
\text { Antisense: 5'-ACT CAG ACA CGG TTC CGT AA-3' } \\
\text { Internal primers: 5'-TCG TAT TAC AAA CCG CGG AGA GGA T-3' } \\
\text { Antisense: 5'-TAC GGT GTT ATT AGT GGA AGT G-3' }\end{array}$ & 1 \\
\hline
\end{tabular}

Abbreviations: gB, glycoprotein B; gN, glycoprotein N; gH, glycoprotein H; HMA-SSCP, heteroduplex mobility assay single-stranded conformation polymorphism; PCR, polymerase chain reaction; RFLP, restriction fragment length polymorphism. 
CMV Genotype in Infants Hu et al. 173

Table 2 Distribution of CMV genotypes and clinical features in infants with symptomatic congenital CMV infection

\begin{tabular}{|c|c|c|c|c|c|c|c|c|}
\hline \multicolumn{9}{|c|}{ Symptomatic congenital CMV infection (moderately to severely symptomatic CMV disease) } \\
\hline \multirow[t]{2}{*}{ No } & \multirow[t]{2}{*}{ Gender } & \multirow[t]{2}{*}{ Clinical manifestation } & \multirow[t]{2}{*}{ Diagnosis } & \multirow[t]{2}{*}{ Specimen type } & \multicolumn{4}{|c|}{ Genotype } \\
\hline & & & & & $g B$ & $\mathrm{gN}$ & $\mathrm{gH}$ & UL144 \\
\hline 1 & $\mathrm{~F}$ & $\begin{array}{l}\text { Intrauterine growth restriction, } \\
\text { respiratory distress, raised levels of } \\
\text { conjugated hyperbilirubinemia }\end{array}$ & PCR & Urine & $\mathrm{gB} 1$ & gN1 & $\mathrm{gH} 1$ & UL144-A \\
\hline 2 & $\mathrm{~F}$ & $\begin{array}{l}\text { Pneumonia, thrombocytopenia, } \\
\text { anemia, hepatosplenomegaly }\end{array}$ & PCR & Urine & $\mathrm{gB} 1$ & $\mathrm{gN2}$ & $\mathrm{gH} 1$ & UL144-A \\
\hline 3 & $M$ & $\begin{array}{l}\text { Sepsis-like, intrauterine growth re- } \\
\text { striction, hepatitis }\end{array}$ & PCR & Urine & $\mathrm{gB1}$ & gN2 & $\mathrm{gH} 2$ & UL144-B \\
\hline 4 & $\mathrm{~F}$ & $\begin{array}{l}\text { Respiratory distress, anemia, } \\
\text { hepatosplenomegaly }\end{array}$ & PCR & Urine, blood & $\mathrm{gB} 2$ & $\mathrm{gN} 4$ & $\mathrm{gH} 1$ & UL144-A \\
\hline 5 & $\mathrm{M}$ & $\begin{array}{l}\text { Respiratory distress, raised levels of } \\
\text { conjugated hyperbilirubinemia, } \\
\text { thrombocytopenia }\end{array}$ & PCR, culture & Urine & $\mathrm{gB} 2$ & gN3 & $\mathrm{gH} 2$ & UL144-A \\
\hline 6 & M & $\begin{array}{l}\text { Sepsis-like, respiratory distress, } \\
\text { anoxic brain damage, raised levels } \\
\text { of conjugated hyperbilirubinemia }\end{array}$ & PCR, culture & Urine & $\mathrm{gB} 3$ & $\mathrm{gN} 4$ & $\mathrm{gH} 1$ & UL144-A \\
\hline 7 & $\mathrm{~F}$ & $\begin{array}{l}\text { Sepsis-like, respiratory distress, } \\
\text { pneumonia, anemia }\end{array}$ & PCR & Urine & $\mathrm{gB3}$ & $\mathrm{gN} 4$ & $\mathrm{gH} 1$ & UL144-A \\
\hline 8 & $M$ & $\begin{array}{l}\text { Respiratory distress, anemia, } \\
\text { hepatosplenomegaly }\end{array}$ & PCR & Urine & $\mathrm{gB} 1$ & gN3 & $\mathrm{gH} 2$ & UL144-C \\
\hline 9 & $\mathrm{~F}$ & $\begin{array}{l}\text { Respiratory distress, raised levels of } \\
\text { conjugated hyperbilirubinemia, in- } \\
\text { trauterine growth restriction }\end{array}$ & PCR & Urine & $\mathrm{gB} 1$ & gN4 & $\mathrm{gH} 1$ & UL144-A \\
\hline 10 & $\mathrm{~F}$ & $\begin{array}{l}\text { Sepsis-like, respiratory distress, } \\
\text { raised levels of conjugated } \\
\text { hyperbilirubinemia }\end{array}$ & PCR & Urine & $\mathrm{gB} 1$ & gN1 & $\mathrm{gH} 1$ & UL144-A \\
\hline 11 & M & $\begin{array}{l}\text { Neutropenia, thrombocytopenia, } \\
\text { anemia, hepatosplenomegaly }\end{array}$ & PCR & Urine & $\mathrm{gB} 2$ & $\mathrm{gN} 4$ & $\mathrm{gH} 1$ & UL144-B \\
\hline 12 & $\mathrm{M}$ & Neurologic abnormalities & $\begin{array}{l}\text { Serodiagnosis, } \\
\text { PCR }\end{array}$ & Blood, CSF & $\mathrm{gB1}$ & $\mathrm{gN2}$ & $\mathrm{gH} 1$ & UL144-C \\
\hline 13 & $\mathrm{~F}$ & $\begin{array}{l}\text { Pneumonia, hepatosplenomegaly, } \\
\text { raised levels of aminotransferase }\end{array}$ & PCR & Urine & $\mathrm{gB} 1$ & gN4 & $\mathrm{gH} 1$ & UL144-B \\
\hline 14 & $\mathrm{M}$ & $\begin{array}{l}\text { Hepatitis, anemia, } \\
\text { hepatosplenomegaly }\end{array}$ & PCR & Urine & $\mathrm{gB} 2$ & $\mathrm{gN} 4$ & $\mathrm{gH} 1$ & UL144-B \\
\hline 15 & $\mathrm{M}$ & $\begin{array}{l}\text { Respiratory distress, thrombocyto- } \\
\text { penia, anemia, } \\
\text { hepatosplenomegaly }\end{array}$ & PCR & Urine & $\mathrm{gB} 1$ & $\mathrm{gN} 4$ & $\mathrm{gH} 2$ & UL144-A \\
\hline 16 & M & $\begin{array}{l}\text { Respiratory distress, intrauterine } \\
\text { growth restriction, hepatitis }\end{array}$ & PCR & Urine & $\mathrm{gB} 1$ & gN4 & $\mathrm{gH} 1$ & UL144-A \\
\hline 17 & $\mathrm{M}$ & Neurologic abnormalities & PCR & CSF, urine & $\mathrm{gB} 2$ & gN3 & $\mathrm{gH} 1$ & UL144-C \\
\hline 18 & $\mathrm{~F}$ & $\begin{array}{l}\text { Sepsis-like, respiratory distress, } \\
\text { anemia, hepatosplenomegaly }\end{array}$ & PCR, culture & Urine & $\mathrm{gB1}$ & gN1 & $\mathrm{gH} 1$ & UL144-A \\
\hline 19 & $\mathrm{~F}$ & $\begin{array}{l}\text { Intracranial hemorrhage, pneumo- } \\
\text { nia, anemia, raised levels of } \\
\text { aminotransferase }\end{array}$ & PCR & Urine & $\mathrm{gB1}$ & gN4 & $\mathrm{gH} 1$ & UL144-B \\
\hline 20 & $M$ & $\begin{array}{l}\text { Sepsis-like, respiratory distress, } \\
\text { hemangioma }\end{array}$ & PCR & Urine & $\mathrm{gB} 2$ & $\mathrm{gN} 4$ & $\mathrm{gH} 1$ & UL144-A \\
\hline 21 & $\mathrm{~F}$ & $\begin{array}{l}\text { Respiratory distress, anoxic brain } \\
\text { damage, pneumonia, anemia }\end{array}$ & PCR & Urine & $\mathrm{gB1}$ & gN4 & $\mathrm{gH} 1$ & UL144-A \\
\hline 22 & $\mathrm{M}$ & $\begin{array}{l}\text { Respiratory distress, raised levels of } \\
\text { conjugated hyperbilirubinemia, } \\
\text { chorioretinitis }\end{array}$ & PCR & Urine & $\mathrm{gB3}$ & $\mathrm{gN4}$ & $\mathrm{gH} 1$ & UL144-A \\
\hline
\end{tabular}


Table 2 (Continued)

\begin{tabular}{|c|c|c|c|c|c|c|c|c|}
\hline \multicolumn{9}{|c|}{ Symptomatic congenital CMV infection (moderately to severely symptomatic CMV disease) } \\
\hline \multirow[t]{2}{*}{ No } & \multirow[t]{2}{*}{ Gender } & \multirow[t]{2}{*}{ Clinical manifestation } & \multirow[t]{2}{*}{ Diagnosis } & \multirow[t]{2}{*}{ Specimen type } & \multicolumn{4}{|c|}{ Genotype } \\
\hline & & & & & $g B$ & $\mathrm{gN}$ & $\mathrm{gH}$ & UL144 \\
\hline 23 & $\mathrm{M}$ & $\begin{array}{l}\text { Sepsis-like, respiratory distress, } \\
\text { cholestasis, cardiomegaly, anemia }\end{array}$ & PCR, culture & Urine & $g B 3$ & gN4 & $\mathrm{gH} 2$ & UL144-B \\
\hline 24 & $\mathrm{~F}$ & $\begin{array}{l}\text { Raised levels of conjugated hyper- } \\
\text { bilirubinemia, raised levels of ala- } \\
\text { nine aminotransferase }\end{array}$ & PCR & Urine, blood & gB1 & gN3 & $\mathrm{gH} 1$ & UL144-A \\
\hline 25 & $\mathrm{~F}$ & $\begin{array}{l}\text { Sepsis-like, thrombocytopenia, } \\
\text { anemia, hepatosplenomegaly }\end{array}$ & PCR & Urine, blood & $\mathrm{gB1}$ & gN4 & $\mathrm{gH} 2$ & UL144-B \\
\hline 26 & $\mathrm{~F}$ & $\begin{array}{l}\text { Respiratory distress, raised levels of } \\
\text { conjugated hyperbilirubinemia, } \\
\text { thrombocytopenia }\end{array}$ & PCR & Urine & $\mathrm{gB1}$ & $\mathrm{gN2}$ & $\mathrm{gH} 1$ & UL144-A \\
\hline 27 & $M$ & $\begin{array}{l}\text { Sepsis-like, respiratory distress, } \\
\text { raised levels of conjugated } \\
\text { hyperbilirubinemia }\end{array}$ & PCR & Urine & $g B 1$ & gN1 & $\mathrm{gH} 1$ & UL144-B \\
\hline
\end{tabular}

Abbreviations: CMV, cytomegalovirus; CSF, cerebrospinal fluid; gB, glycoprotein B; gN, glycoprotein N; gH, glycoprotein H; PCR, polymerase chain reaction.

symptoms and asymptomatic infants were not included in the analysis. A $p$-value of less than 0.05 was considered statistically significant.

\section{Results}

Fifty immunocompetent infants with symptomatic congenital CMV infection were analyzed. Twenty-seven were diagnosed with moderate to severe symptomatic CMV disease, and the remainder were diagnosed with mild symptomatic CMV disease. Further, 25 infants with asymptomatic CMV infection were served as a baseline for direct comparison. Baseline characteristics, laboratory data, and genotypes of CMV are summarized in - Tables 2 and 3.

The incidence of gB genotypes in symptomatic neonates was gB1, 46.0\%, 23/50; gB2, 30.0\%, 15/50; and gB3, 24.0\%, 12/50. Notably, a significantly higher incidence of gB1 (63.0\%, $17 / 27$ ) was observed in moderate to severe CMV infections than in mild infections (chi-square $=6.918, p=0.031$ ) (-Table 4).

The overall incidence of individual genotypes in the study cohort was gN1, 22.0\%, 11/50; gN2, 8.0\%, 4/50; gN3, 26.0\%, $13 / 50$; and gN4, 44.0\%, 22/50. gN1 (14.8\%, 4/27) and gN3 $(14.8 \%, 4 / 27)$ were less prevalent in moderate to severe CMV disease $($ chi-square $=14.278, p=0.003)(-$ Table 4$)$.

gH1 and gH2 genotypes were found in 70.0\% (35/50) and $30.0 \%$ (15/50) of infants, respectively. The prevalence of $\mathrm{gH}$ genotypes between symptomatic and asymptomatic infants was not statistically significant, and these genotypes were correlated with disease severity (-Table 4 ).

The incidence of UL144 genotypes in patients with symptomatic infection was UL144-A, 52.0\%, 26/50; UL144-B, 40.0\%, 20/50; and UL144-C, 8.0\%, 4/50. Significantly greater prevalence $(68.0 \%, 17 / 25)$ of UL144-B was observed in asymptomatic infants (chi-square $=6.119$, $p=0.047$ ) (-Table 4).
The gN1 $(p=0.010)$ and UL144-B $(p=0.034)$ genotypes were associated with a reduced risk of developing symptomatic congenital CMV infection. Similarly, the gN1 $(p=0.020)$ and gN3 $(p=0.022)$ genotypes were associated with a reduced risk of severe symptomatic disease. Conversely, gB1 $(p=0.018)$ was the most virulent genotype, associated with severe manifestations in congenital CMV infection (-Table 5).

\section{Discussion}

$\mathrm{gB}$ is a polyprotein cleaved at position 460 within a cleavage zone (CLZ). Its variability is produced by variation and possible recombination at the CLZ and N-terminal region. ${ }^{14}$ The four gB genotypes currently identified reflect alterations in the CLZ region. ${ }^{14}$ Our study shows that the prevalence of gB genotypes in symptomatic and asymptomatic infants is not different. In contrast, a relationship exists between gB genotypes and outcome; gB1 variants are associated with more severe infection. Critical gB-specific nonneutralizing antibodies exhibit genotype-specific gB recognition on the cell surface. This group of nonneutralizing gB-specific monoclonal antibodies typically bind preferentially to gB2 and gB4. ${ }^{15}$ We speculate that gB1producing CMV strains may elicit a severe immunopathological response that leads to tissue damage and disease manifestations.

Previous studies demonstrated that CMV gN1 and gN3 variants are less virulent genotypes than gN2 and gN4 and are associated with a reduced risk of some symptoms. ${ }^{16-18}$ Our results confirmed that the gN1 and gN3 variants are less virulent and demonstrate that both genotypes might display a decreased risk of severe symptomatic CMV disease. The gN1 genotype is a typical AD169-like glycoprotein that is immunologically separate from other CMV clinical genotypes. ${ }^{16,17,19}$ The obtained results suggest that the gN1 
CMV Genotype in Infants $\mathrm{Hu}$ et al. 175

Table 3 Distribution of CMV genotypes in infants with symptomatic and asymptomatic congenital CMV infections

\begin{tabular}{|c|c|c|c|c|c|c|c|c|c|c|c|c|}
\hline \multicolumn{13}{|c|}{ Group settings } \\
\hline \multicolumn{7}{|c|}{ Symptomatic congenital CMV infection (mildly symptomatic CMV disease) } & \multicolumn{6}{|c|}{ Asymptomatic congenital CMV infection } \\
\hline \multirow[t]{2}{*}{ No } & \multirow[t]{2}{*}{ Gender } & \multirow[t]{2}{*}{ Clinical manifestation } & \multicolumn{4}{|c|}{ Genotype } & \multirow[t]{2}{*}{ No } & \multirow[t]{2}{*}{ Gender } & \multicolumn{4}{|c|}{ Genotype } \\
\hline & & & $\mathrm{gB}$ & $\mathrm{gN}$ & $\mathrm{gH}$ & UL144 & & & $\mathrm{gB}$ & $\mathrm{gN}$ & $\mathrm{gH}$ & UL144 \\
\hline 1 & M & $\begin{array}{l}\text { Raised levels of conjugated } \\
\text { hyperbilirubinemia }\end{array}$ & $\mathrm{gB2}$ & gN4 & $\mathrm{gH} 1$ & UL144-A & 1 & $\mathrm{~F}$ & gB1 & gN1 & $\mathrm{gH} 2$ & UL144-B \\
\hline 2 & $\mathrm{M}$ & $\begin{array}{l}\text { Raised levels of conjugated } \\
\text { hyperbilirubinemia }\end{array}$ & $\mathrm{gB} 2$ & gN3 & $\mathrm{gH} 1$ & UL144-B & 2 & $\mathrm{M}$ & $\mathrm{gB3}$ & gN1 & $\mathrm{gH} 1$ & UL144-A \\
\hline 3 & $M$ & Hepatomegaly & $g B 1$ & $\mathrm{gN} 3$ & $\mathrm{gH} 2$ & UL144-B & 3 & $\mathrm{M}$ & $\mathrm{gB3}$ & gN4 & $\mathrm{gH} 1$ & UL144-B \\
\hline 4 & $M$ & $\begin{array}{l}\text { Raised levels of alanine } \\
\text { aminotransferase }\end{array}$ & $\mathrm{gB3}$ & gN1 & $\mathrm{gH} 2$ & UL144-A & 4 & $M$ & $\mathrm{gB1}$ & gN4 & $\mathrm{gH} 1$ & UL144-A \\
\hline 5 & $\mathrm{~F}$ & Low platelet count & $g B 1$ & $\mathrm{gN} 4$ & $\mathrm{gH} 2$ & UL144-C & 5 & $\mathrm{~F}$ & $\mathrm{gB1}$ & gN3 & $\mathrm{gH} 2$ & UL144-B \\
\hline 6 & $\mathrm{M}$ & Neutropenia & $\mathrm{gB1}$ & $\mathrm{gN} 4$ & $\mathrm{gH} 1$ & UL144-B & 6 & $M$ & $g B 1$ & gN1 & $\mathrm{gH} 2$ & UL144-B \\
\hline 7 & $\mathrm{~F}$ & Hepatomegaly & $\mathrm{gB} 2$ & $\mathrm{gN} 3$ & $\mathrm{gH} 1$ & UL144-B & 7 & $\mathrm{M}$ & $g B 2$ & gN3 & $\mathrm{gH} 2$ & UL144-A \\
\hline 8 & $\mathrm{~F}$ & $\begin{array}{l}\text { Raised levels of alanine } \\
\text { aminotransferase }\end{array}$ & $\mathrm{gB3}$ & $\mathrm{gN4}$ & $\mathrm{gH} 1$ & UL144-A & 8 & $\mathrm{~F}$ & $\mathrm{gB2}$ & gN3 & $\mathrm{gH} 1$ & UL144-A \\
\hline 9 & $\mathrm{~F}$ & Hepatosplenomegaly & gB3 & gN3 & $\mathrm{gH} 2$ & UL144-A & 9 & $\mathrm{~F}$ & $\mathrm{gB3}$ & gN1 & $\mathrm{gH} 1$ & UL144-B \\
\hline 10 & $\mathrm{M}$ & Low platelet count & $\mathrm{gB} 2$ & gN1 & $\mathrm{gH} 1$ & UL144-B & 10 & $\mathrm{~F}$ & $\mathrm{gB} 2$ & gN1 & $\mathrm{gH} 1$ & UL144-B \\
\hline 11 & $\mathrm{~F}$ & Low platelet count & $\mathrm{gB3}$ & $\mathrm{gN} 3$ & $\mathrm{gH} 2$ & UL144-A & 11 & $\mathrm{M}$ & gB3 & gN3 & $\mathrm{gH} 1$ & UL144-B \\
\hline 12 & $\mathrm{M}$ & $\begin{array}{l}\text { Raised levels of alanine } \\
\text { aminotransferase }\end{array}$ & gB1 & gN1 & $\mathrm{gH} 1$ & UL144-B & 12 & $\mathrm{~F}$ & $\mathrm{gB1}$ & gN1 & $\mathrm{gH} 1$ & UL144-B \\
\hline 13 & $M$ & $\begin{array}{l}\text { Raised levels of conjugated } \\
\text { hyperbilirubinemia }\end{array}$ & $\mathrm{gB} 2$ & gN1 & $\mathrm{gH} 1$ & UL144-A & 13 & $\mathrm{M}$ & $\mathrm{gB3}$ & gN4 & $\mathrm{gH} 1$ & UL144-A \\
\hline 14 & $M$ & $\begin{array}{l}\text { Raised levels of alanine } \\
\text { aminotransferase }\end{array}$ & gB3 & gN1 & $\mathrm{gH} 2$ & UL144-B & 14 & $M$ & $\mathrm{gB1}$ & gN1 & $\mathrm{gH} 2$ & UL144-B \\
\hline 15 & $\mathrm{~F}$ & $\begin{array}{l}\text { Raised levels of alanine } \\
\text { aminotransferase }\end{array}$ & $\mathrm{gB3}$ & gN1 & $\mathrm{gH} 2$ & UL144-A & 15 & $\mathrm{~F}$ & $\mathrm{gB1}$ & gN3 & $\mathrm{gH} 1$ & UL144-B \\
\hline 16 & $\mathrm{~F}$ & Neutropenia & $g B 2$ & $\mathrm{gN} 4$ & $\mathrm{gH} 1$ & UL144-B & 16 & $\mathrm{~F}$ & $\mathrm{gB} 1$ & gN3 & $\mathrm{gH} 1$ & UL144-B \\
\hline 17 & $\mathrm{~F}$ & Low platelet count & $g B 2$ & gN1 & $\mathrm{gH} 2$ & UL144-A & 17 & $\mathrm{~F}$ & $\mathrm{gB2}$ & gN1 & $\mathrm{gH} 1$ & UL144-B \\
\hline 18 & M & $\begin{array}{l}\text { Raised levels of conjugated } \\
\text { hyperbilirubinemia }\end{array}$ & $g B 2$ & $\mathrm{gN} 4$ & $\mathrm{gH} 1$ & UL144-B & 18 & $\mathrm{~F}$ & $\mathrm{gB1}$ & gN1 & $\mathrm{gH} 2$ & UL144-A \\
\hline 19 & $\mathrm{~F}$ & Anemia & $\mathrm{gB3}$ & $\mathrm{gN} 3$ & $\mathrm{gH} 2$ & UL144-B & 19 & $\mathrm{~F}$ & $g B 1$ & $\mathrm{gN} 4$ & $\mathrm{gH} 2$ & UL144-B \\
\hline 20 & $\mathrm{~F}$ & $\begin{array}{l}\text { Raised levels of alanine } \\
\text { aminotransferase }\end{array}$ & gB1 & gN3 & $\mathrm{gH} 1$ & UL144-A & 20 & $\mathrm{M}$ & $\mathrm{gB3}$ & gN1 & $\mathrm{gH} 1$ & UL144-B \\
\hline 21 & $M$ & $\begin{array}{l}\text { Raised levels of alanine } \\
\text { aminotransferase }\end{array}$ & $\mathrm{gB} 3$ & gN3 & $\mathrm{gH} 1$ & UL144-B & 21 & $\mathrm{M}$ & $\mathrm{gB} 1$ & gN3 & $\mathrm{gH} 2$ & UL144-A \\
\hline 22 & $\mathrm{M}$ & Hepatosplenomegaly & $\mathrm{gB} 2$ & $\mathrm{gN} 4$ & $\mathrm{gH} 1$ & UL144-B & 22 & $\mathrm{~F}$ & $g B 2$ & gN3 & $\mathrm{gH} 1$ & UL144-B \\
\hline 23 & $M$ & Hepatomegaly & $\mathrm{gB1}$ & $\mathrm{gN} 1$ & $\mathrm{gH} 1$ & UL144-A & 23 & $\mathrm{~F}$ & $g B 1$ & $\mathrm{gN} 4$ & $\mathrm{gH} 2$ & UL144-B \\
\hline & & & & & & & 24 & $M$ & $\mathrm{gB3}$ & gN1 & $\mathrm{gH} 1$ & UL144-A \\
\hline & & & & & & & 25 & $\mathrm{M}$ & $\mathrm{gB1}$ & gN1 & $\mathrm{gH} 1$ & UL144-B \\
\hline
\end{tabular}

Abbreviations: CMV, cytomegalovirus; gB, glycoprotein B; gN, glycoprotein N; gH, glycoprotein $\mathrm{H}$.

genotype of CMV might be an important virological marker of asymptomatic infection in infants.

Nahar et $\mathrm{al}^{3}$ described that the $\mathrm{gH} 2$ variant of CMV was significantly more prevalent than the gH1 variant in ulcerative colitis active patients with severe and moderate symptoms, which possibly indicates more virulence. Another study has demonstrated that gH1 represents the more virulent genotype, compared with $\mathrm{gH} 2$, and is associated with SNHL. However, a virulence difference between these genotypes is not observed for other clinical symptoms. ${ }^{20}$ No genotypes of gH in the present study are confirmed to be associated with virulence. At least two explanations are possible: (1) a specific CMV genotype may show strong virulence in some CMV-related diseases, while in other CMV-related diseases or asymptomatic infants, it may not show corresponding virulence 
Table 4 Distribution of CMV genotypes in moderately to severely infection and mildly infection among infants infected congenitally

\begin{tabular}{|c|c|c|c|c|c|c|c|c|c|}
\hline Group & gB1 & \multicolumn{2}{|l|}{$\mathrm{gB} 2$} & $\mathrm{gB3}$ & Total & \multirow[t]{2}{*}{ Chi-square } & \multirow[t]{2}{*}{$p$-Value } & \multirow[t]{3}{*}{ Chi-square } & \multirow[t]{3}{*}{$p$-Value } \\
\hline Asymptomatic, n (\%) & $13(52.0)$ & \multicolumn{2}{|l|}{$5(20.0)$} & $7(28.0)$ & 25 & & & & \\
\hline Symptomatic, $n$ (\%) & $23(46.0)$ & \multicolumn{2}{|l|}{$15(30.0)$} & $12(24.0)$ & 50 & 0.855 & 0.652 & & \\
\hline $\begin{array}{l}\text { Moderate to severe } \\
\text { CMV infection, } n(\%)\end{array}$ & $17(63.0)$ & \multicolumn{2}{|l|}{$6(22.2)$} & $4(14.8)$ & 27 & 1.368 & 0.505 & \multirow[t]{2}{*}{6.918} & \multirow[t]{2}{*}{0.031} \\
\hline $\begin{array}{l}\text { Mild CMV } \\
\text { infection, } n(\%)\end{array}$ & $6(26.1)$ & \multicolumn{2}{|l|}{$9(39.1)$} & $8(34.8)$ & 23 & 3.712 & 0.156 & & \\
\hline Group & gN1 & gN2 & gN3 & gN4 & Total & \multirow[t]{2}{*}{ Chi-square } & \multirow[t]{2}{*}{$p$-Value } & \multirow[t]{3}{*}{ Chi-square } & \multirow[t]{3}{*}{$p$-Value } \\
\hline Asymptomatic, $n(\%)$ & $12(48.0)$ & $0(0)$ & $8(32.0)$ & $5(20.0)$ & 25 & & & & \\
\hline Symptomatic, n (\%) & $11(22.0)$ & $4(8.0)$ & $13(26.0)$ & $22(44.0)$ & 50 & 8.555 & 0.036 & & \\
\hline $\begin{array}{l}\text { Moderate to } \\
\text { severe CMV } \\
\text { infection, } n(\%)\end{array}$ & $4(14.8)$ & $4(14.8)$ & $4(14.8)$ & $15(55.6)$ & 27 & 14.278 & 0.003 & \multirow[t]{2}{*}{9.390} & \multirow[t]{2}{*}{0.025} \\
\hline $\begin{array}{l}\text { Mild CMV } \\
\text { infection, } n(\%)\end{array}$ & $7(30.4)$ & $0(0)$ & $9(39.1)$ & $7(30.4)$ & 23 & 1.627 & 0.443 & & \\
\hline Group & \multicolumn{2}{|l|}{$\mathrm{gH} 1$} & \multicolumn{2}{|l|}{$\mathrm{gH} 2$} & Total & \multirow[t]{2}{*}{ Chi-square } & \multirow[t]{2}{*}{$p$-Value } & \multirow[t]{3}{*}{ Chi-square } & \multirow[t]{3}{*}{ p-Value } \\
\hline Asymptomatic, $n(\%)$ & \multicolumn{2}{|l|}{$16(64.0)$} & \multicolumn{2}{|l|}{$9(36.0)$} & 25 & & & & \\
\hline Symptomatic, $n(\%)$ & \multicolumn{2}{|l|}{$35(70.0)$} & \multicolumn{2}{|l|}{$15(30.0)$} & 50 & 0.276 & 0.600 & & \\
\hline $\begin{array}{l}\text { Moderate to severe } \\
\text { CMV infection, } n(\%)\end{array}$ & \multicolumn{2}{|l|}{$21(77.8)$} & \multicolumn{2}{|l|}{$6(22.2)$} & 27 & 1.201 & 0.273 & \multirow[t]{2}{*}{1.691} & \multirow[t]{2}{*}{0.193} \\
\hline $\begin{array}{l}\text { Mild CMV } \\
\text { infection, } n(\%)\end{array}$ & \multicolumn{2}{|l|}{$14(60.9)$} & \multicolumn{2}{|l|}{$9(39.1)$} & 23 & 0.050 & 0.823 & & \\
\hline Group & UL144-A & \multicolumn{2}{|l|}{ UL144-B } & UL144-C & Total & Chi-square & $p$-Value & Chi-square & $p$-Value \\
\hline Asymptomatic, n (\%) & $8(32.0)$ & $17(68.0)$ & & $0(0)$ & 25 & & & & \\
\hline Symptomatic, n (\%) & $26(52.0)$ & $20(40.0)$ & & $4(8.0)$ & 50 & 6.119 & 0.047 & & \\
\hline $\begin{array}{l}\text { Moderate to severe } \\
\text { CMV infection, } n(\%)\end{array}$ & $16(59.3)$ & $8(29.6)$ & & $3(11.3)$ & 27 & 8.843 & 0.012 & 2.883 & 0.237 \\
\hline $\begin{array}{l}\text { Mild CMV } \\
\text { infection, } n(\%)\end{array}$ & $10(43.5)$ & $12(52.2)$ & & $1(4.3)$ & 23 & 2.004 & 0.367 & & \\
\hline
\end{tabular}

Abbreviations: CMV, cytomegalovirus; gB, glycoprotein B; gN, glycoprotein N; gH, glycoprotein $\mathrm{H}$.

characteristics and (2) study populations may differ. The study population in the earlier reports was from patients with active ulcerative colitis and SNHL. This population differs substantially from the population in the present study.
We also investigated the UL144 gene because the protein has a role in allowing CMV to escape the immune response. Waters et al indicated that detection of UL144-A and UL144$\mathrm{C}$ was associated with severe congenital CMV infection and was more likely to lead to long-term CMV-associated clinical

Table 5 Factors associated with outcome of CMV infection and severity of CMV disease

\begin{tabular}{|c|c|c|c|c|c|}
\hline Factors & Group settings & Genotype & B & $p$-Value & OR (95\% Cl) \\
\hline \multirow[t]{2}{*}{ Outcome of CMV infection ${ }^{a}$} & \multirow{2}{*}{$\begin{array}{l}\text { Symptomatic congenital CMV } \\
\text { infection } \\
\text { Asymptomatic congenital } \\
\text { CMV infection }\end{array}$} & gN1 & -1.720 & 0.010 & $0.179(0.049-0.657)$ \\
\hline & & UI144-B & -1.156 & 0.034 & $0.315(0.108-0.917)$ \\
\hline \multirow[t]{3}{*}{ Severity of CMV disease ${ }^{a}$} & \multirow{3}{*}{$\begin{array}{l}\text { Mildly symptomatic CMV dis- } \\
\text { ease } \\
\text { Moderately to severely symp- } \\
\text { tomatic CMV disease }\end{array}$} & $\mathrm{gB} 1$ & 1.740 & 0.018 & $5.695(1.349-24.048)$ \\
\hline & & gN1 & -2.144 & 0.020 & $0.117(0.019-0.711)$ \\
\hline & & $\mathrm{gN3}$ & -1.985 & 0.022 & $0.137(0.025-0.752)$ \\
\hline
\end{tabular}

Abbreviations: $\mathrm{Cl}$, confidence interval; CMV, cytomegalovirus; gB, glycoprotein B; gN, glycoprotein N; OR, odds ratio.

${ }^{\mathrm{a} B y}$ backward LR binary logistic regression. 
manifestations. ${ }^{21}$ Similarly, Arav-Boger et al showed that UL144-A and UL144-C are related to congenital CMV symptoms, and UL144-C was identified only in symptomatic patients. $^{22}$ Our findings are consistent and further demonstrate that the UL144-B genotype may be associated with asymptomatic congenital infection. CRD1 shows greater variation in UL144-A and UL144-C but is better conserved in UL144-B than the Towne strain. ${ }^{6}$ This finding could be a reason for the enhanced virulence of UL144-A and UL144-C.

Our study has some limitations. Some uncertainty exists in the grouping of disease severity in children. Nine of 27 patients exhibited sepsis-like syndrome. However, other pathogens can cause sepsis-like symptoms, including certain viruses, bacteria, and fungi. Such pathogens were not tested or detected. The existence of coinfection might aggravate clinical symptoms, thus causing errors in grouping and statistical deviation.

\section{Conclusion}

In summary, our study demonstrated a relationship between the gN1 and UL144-B genotypes and asymptomatic congenital CMV infection after birth. Further, detection of the gB1, gN1, or gN3 genotypes may help define this disease's manifestations in children. This study suggests that the gB, gN, and UL144 genotypes may be valuable virological markers of congenital CMV disease. Our data will help predict the severity of congenital CMV infection and help formulate preventive measures.

Conflict of Interest

None declared.

\section{References}

1 Mei C, Yang W, Wei X, Wu K, Huang D. The unique microbiome and innate immunity during pregnancy. Front Immunol 2019; 10:2886

2 Hu H, Peng W, Peng Q Cheng Y. Cytomegalovirus genotype distribution among congenital and perinatal infected patients with CMV-associated thrombocytopenia. Fetal Pediatr Pathol 2020:1-10

3 Nahar S, Hokama A, Iraha A, et al. Distribution of cytomegalovirus genotypes among ulcerative colitis patients in Okinawa, Japan. Intest Res 2018;16(01):90-98

$4 \mathrm{Hu} \mathrm{H}$, Cheng Y, Peng $\mathrm{Q}$ Chen K. Clinical features, treatment courses, and distribution of cytomegalovirus genotypes among thrombocytopenia patients aged younger than 12 months. Am J Perinatol 2020:10

5 Bitra A, Nemčovičová I, Picarda G, et al. Structure of human cytomegalovirus UL144, an HVEM orthologue, bound to the B and T cell lymphocyte attenuator. J Biol Chem 2019;294(27): 10519-10529
6 Guo G, Zhang L, Ye S, et al. Polymorphisms and features of cytomegalovirus UL144 and UL146 in congenitally infected neonates with hepatic involvement. PLoS One 2017;12(02):e0171959

7 Chen HP, Jiang JK, Chan CH, et al. Genetic polymorphisms of the human cytomegalovirus UL144 gene in colorectal cancer and its association with clinical outcome. J Gen Virol 2015;96(12):3613-3623

8 Luck SE, Wieringa JW, Blázquez-Gamero D, et al; ESPID Congenital CMV Group Meeting, Leipzig 2015. Congenital cytomegalovirus: a European expert consensus statement on diagnosis and management. Pediatr Infect Dis J 2017;36(12):1205-1213

9 Rawlinson WD, Boppana SB, Fowler KB, et al. Congenital cytomegalovirus infection in pregnancy and the neonate: consensus recommendations for prevention, diagnosis, and therapy. Lancet Infect Dis 2017;17(06):e177-e188

10 Yu ZS, Zou CC, Zheng JY, Zhao ZY. Cytomegalovirus gB genotype and clinical features in Chinese infants with congenital infections. Intervirology 2006;49(05):281-285

11 Guo S, Yu MM, Li G, Zhou H, Fang F, Shu SN. Studies on genotype of human cytomegalovirus glycoprotein $\mathrm{H}$ from infantile clinical isolates. Zhonghua Er Ke Za Zhi 2013;51(04):260-264

12 Chen HP, Lin JC, Yang SP, et al. The type-2 variant of human cytomegalovirus glycoprotein $\mathrm{N}(\mathrm{gN}-2)$ is not the rarest in the Chinese population of Taiwan: influence of primer design. J Virol Methods 2008;151(01):161-164

13 He R, Ruan Q, Xia C, et al. Sequence variability of human cytomegalovirus UL144 open reading frame in low-passage clinical isolates. Chin Med Sci J 2004;19(04):293-297

14 Grosjean J, Hantz S, Cotin S, et al. Direct genotyping of cytomegalovirus envelope glycoproteins from toddler's saliva samples. J Clin Virol 2009;46(Suppl 4):S43-S48

15 Goodwin ML, Webster HS, Wang HY, et al. Specificity and effector functions of non-neutralizing gB-specific monoclonal antibodies isolated from healthy individuals with human cytomegalovirus infection. Virology 2020;548:182-191

16 Paradowska E, Jabłońska A, Studzińska M, et al. Distribution of cytomegalovirus gN variants and associated clinical sequelae in infants. J Clin Virol 2013;58(01):271-275

17 Pignatelli S, Lazzarotto T, Gatto MR, et al. Cytomegalovirus gN genotypes distribution among congenitally infected newborns and their relationship with symptoms at birth and sequelae. Clin Infect Dis 2010;51(01):33-41

18 Mujtaba G, Khurshid A, Sharif S, et al. Distribution of cytomegalovirus among neonates born to infected mothers in Islamabad, Pakistan. PLoS One 2016;11(07):e0156049

19 Pignatelli S, Rossini G, Dal Monte P, Gatto MR, Landini MP. Human cytomegalovirus glycoprotein $\mathrm{N}$ genotypes in AIDS patients. AIDS 2003;17(05):761-763

20 Paradowska E, Jabłońska A, Studzińska M, et al. Cytomegalovirus glycoprotein $\mathrm{H}$ genotype distribution and the relationship with hearing loss in children. J Med Virol 2014;86(08):1421-1427

21 Waters A, Hassan J, De Gascun C, et al. Human cytomegalovirus UL144 is associated with viremia and infant development sequelae in congenital infection. J Clin Microbiol 2010;48(11):3956-3962

22 Arav-Boger R, Battaglia CA, Lazzarotto T, et al. Cytomegalovirus (CMV)-encoded UL144 (truncated tumor necrosis factor receptor) and outcome of congenital CMV infection. J Infect Dis 2006; 194(04):464-473 